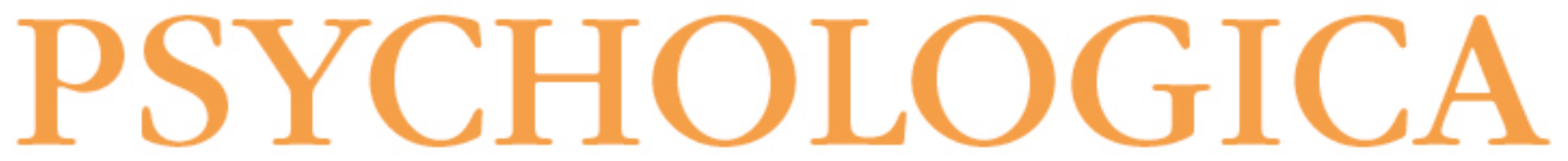

Cuidados paliativos oncológicos em contexto de internamento e domiciliário: necessidades, morbilidade psicológica e luto antecipatório nos familiares do doente terminal e impacto na qualidade de vida familiar

Autor(es): $\quad$ Areia, Neide P.; Major, Sofia; Gaspar, Catarina; Relvas, Ana Paula

Publicado por: Imprensa da Universidade de Coimbra

URL persistente:

URI:http://hdl.handle.net/10316.2/43288

DOI:

DOI:https://doi.org/10.14195/1647-8606_60-2_2

Accessed : $\quad$ 26-Apr-2023 15:45:46

A navegação consulta e descarregamento dos títulos inseridos nas Bibliotecas Digitais UC Digitalis, UC Pombalina e UC Impactum, pressupõem a aceitação plena e sem reservas dos Termos e Condições de Uso destas Bibliotecas Digitais, disponíveis em https://digitalis.uc.pt/pt-pt/termos.

Conforme exposto nos referidos Termos e Condições de Uso, o descarregamento de títulos de acesso restrito requer uma licença válida de autorização devendo o utilizador aceder ao(s) documento(s) a partir de um endereço de IP da instituição detentora da supramencionada licença.

Ao utilizador é apenas permitido o descarregamento para uso pessoal, pelo que o emprego do(s) título(s) descarregado(s) para outro fim, designadamente comercial, carece de autorização do respetivo autor ou editor da obra.

Na medida em que todas as obras da UC Digitalis se encontram protegidas pelo Código do Direito de Autor e Direitos Conexos e demais legislação aplicável, toda a cópia, parcial ou total, deste documento, nos casos em que é legalmente admitida, deverá conter ou fazer-se acompanhar por este aviso. 


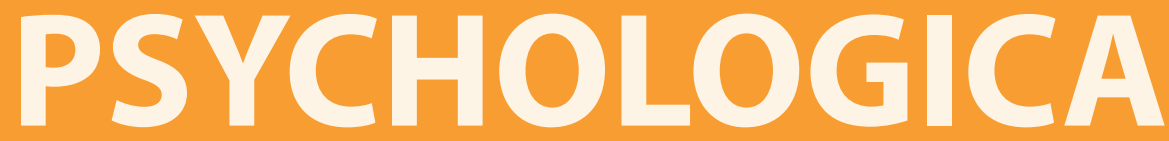




\title{
Cuidados paliativos oncológicos em contexto de internamento e domiciliário: Necessidades, morbilidade psicológica e luto antecipatório nos familiares do doente terminal e impacto na qualidade de vida familiar
}

\author{
Neide P. Areia ${ }^{1}$, Sofia Major², Catarina Gaspar³ e Ana Paula Relvas ${ }^{4}$
}

Palliative oncology in hospice and home care: Needs, psychological morbidity and anticipatory grief on patient's relatives and impact on family quality of life

\begin{abstract}
Terminal cancer has a profound impact on family and its' elements. Therefore, palliative care foresees a family support. However, there are not many studies that aim to determine the family needs within palliative care. The present study aims to determine family needs, psychological morbidity and anticipatory grief of terminal patients' relatives, distinguishing two care contexts (hospice and home care), and examine the influence of unmet needs, anticipatory grief, psychological morbidity and care setting on the perceived family quality of life. For such, an exploratory study was conducted with a sample of 40 terminal cancer patients' relatives in palliative care. It was found that psychosocial needs are lesser satisfied in
\end{abstract}

\footnotetext{
1 Bolseira de Doutoramento FCT: SFRH/BD/86178/2012, Faculdade de Psicologia e de Ciências da Educação, Universidade de Coimbra. Email: neideareia@hotmail.com

2 Professora Auxiliar, Faculdade de Ciências Sociais e Humanas, Universidade dos Açores e Investigadora do Centro de Estudos Sociais (CES) da Universidade de Coimbra. Email: smajor@fpce.uc.pt

3 Mestre em Psicologia Clínica. Email: cheira_246@hotmail.com

4 Professora Catedrática da Faculdade de Psicologia e de Ciências da Educação, Universidade de Coimbra e Investigadora do Centro de Estudos Sociais (CES) da Universidade de Coimbra. Email: aprelvas@fpce.uc.pt
} 
home-based palliative care. Regardless the context of care, patients' relatives report clinically significant levels of psychological morbidity and anticipatory grief with impact on family perceived quality of life. Unmet needs, psychological morbidity and anticipatory grief seem to influence on the perceived family quality of life. This study demonstrates the importance of attending patients' relatives in palliative care, in a holistic and multidisciplinary approach.

Keywords: terminal cancer; palliative care; family; needs, psychological morbidity; anticipatory grief

\section{Resumo}

A doença oncológica na fase terminal tem um importante impacto na família e seus elementos. Por este motivo, os cuidados paliativos pressupõem o suporte à família. No entanto, são escassos os estudos que visam determinar as necessidades das famílias em cuidados paliativos. O presente estudo teve como objetivos determinar quais as necessidades dos familiares dos doentes terminais, a presença de morbilidade psicológica e experiência de luto antecipatório, distinguindo dois contextos de prestação de cuidados (internamento e domicílio), e examinar a influência de uma satisfação inadequada das necessidades, luto antecipatório e contexto de prestação de cuidados na perceção de qualidade de vida familiar. Para tal, procedeu-se a um estudo exploratório junto de uma amostra de 40 familiares de doentes oncológicos terminais em cuidados paliativos. Verificou-se que as necessidades de suporte psicossocial são menos satisfeitas em contexto de cuidados domiciliários. Independentemente do contexto de prestação de cuidados, os familiares apresentam níveis clinicamente significativos de morbilidade psicológica e luto antecipatório com impacto na perceção da qualidade de vida familiar. A satisfação inadequada das necessidades, a presença de morbilidade psicológica e a experiência de luto antecipatório influenciam a perceção de qualidade de vida familiar. Este estudo vem demonstrar a importância de atender às necessidades dos familiares em cuidados paliativos, numa perspetiva holística e multidisciplinar.

Palavras-chave: doença oncológica terminal; cuidados paliativos; família; necessidades, morbilidade psicológica; luto antecipatório.

\section{INTRODUÇÃO}

O cancro é um problema de saúde pública (Siegel, Miller, \& Jemal, 2016) e, por isso, considerado por alguns autores uma epidemia moderna (Simões, 2014). O número de novos casos por ano tem aumentado significativamente em Portugal 
e no mundo (World Health Organization [WHO], 2015). No panorama internacional, espera-se que a incidência das doenças oncológicas aumente em $70 \%$ nas próximas duas décadas (WHO, 2015). Apesar da taxa de mortalidade por tumor maligno ter vindo a diminuir discretamente (Direção Geral de Saúde [DGS], 2014), o cancro continua a ser a segunda causa de morte em Portugal (Instituto Nacional de Estatística [INE], 2015). Cerca de 25\% dos óbitos registados são derivados à doença oncológica (INE, 2013).

Dada a elevada mortalidade associada ao cancro, continua a ser relevante e pertinente implementar e desenvolver programas de cuidados paliativos que respondam adequada e satisfatoriamente às necessidades dos doentes terminais e suas famílias (Osswald, 2013). Os cuidados paliativos assumem-se como a abordagem privilegiada de cuidados quando a doença entra numa fase de evolução avançada e irreversível e pressupõe o alívio do sofrimento do doente, através de um rigoroso controlo da sintomatologia decorrente da sua deterioração física e aproximação da morte; a garantia da qualidade de vida, bem-estar e conforto; um acompanhamento multidisciplinar; e o suporte à família quer antes, quer após a ocorrência da morte (WHO, 2002).

Embora, na maioria das vezes, os doentes prefiram receber cuidados e morrer em casa (Cooper, 2014; European Association for Palliative Care [EAPC], 2010), os cuidados paliativos, aos doentes terminais, podem ser prestados em unidades de internamento ou no domicílio, geralmente em casa do doente. O doente é internado quando: a severidade da sintomatologia exige cuidados intensos, especializados e complexos, com o objetivo de estabilizar o estado funcional do doente; se verificam situações de abandono ou negligência, distúrbios emocionais graves e níveis elevados de sobrecarga, exaustão física e emocional nos cuidadores ou claudicação familiar (EAPC, 2010). Apesar de pouco explorado na literatura, sabe-se que o contexto de internamento apresenta desafios específicos às famílias. São particularmente frequentes: a) perda de privacidade e intimidade, o que pode dificultar a resolução do luto antecipatório e post mortem; b) aumento de dificuldades na comunicação e conflitos entre profissionais de saúde e família; e c) desenvolvimento de fenómenos de exaustão física e emocional, burnout e dificuldades económicas (Ferrel, Borneman, \& Thai, 2009).

No entanto, sempre que possível, é desejável atender ao desejo do doente de permanecer em casa e, neste caso, os cuidados especializados são prestados em ambulatório ou no domicílio por uma equipa de cuidados paliativos (DGS, 2004; EAPC, 2010). Os restantes cuidados são levados a cabo pela família que, em contexto de cuidados paliativos domiciliários, se assume como uma inestimável parceira dos profissionais de saúde na garantia do conforto e bem-estar do doente (Stajduhar \& Cohen, 2009). Cuidar do doente em casa é particu- 
larmente desafiante para os familiares que assumem o papel de prestação de cuidados. É muito frequente os cuidadores isolarem-se socialmente, experienciarem elevados níveis de morbilidade psicológica (e.g., ansiedade, depressão), sobrecarga e burnout, problemas de saúde e um elevado risco de mortalidade (e.g., hipertensão, distúrbios do sono) e dificuldades económicas (Stajduhar \& Cohen, 2009).

Independentemente do contexto de prestação de cuidados, é indubitável: a doença, o morrer e a morte não são acontecimentos meramente individuais ou restritos, mas de significado e impacto coletivo (Osswald, 2013). A fase terminal da doença e, por conseguinte, a eminência da morte de um dos membros da família assume-se como uma das crises mais difíceis que o sistema deve enfrentar, com impactos no sistema no seu todo, seus elementos e relações (Monroe \& Oliviere, 2009; Rolland, 1984, 1987, 2005). Na família e seus elementos podem verificar-se: a) alterações na dinâmica de organização e funcionamento familiar (e.g., reajustamento da estrutura familiar, redistribuição de papéis); b) alterações nos padrões de comunicação (e.g., conspiração do silêncio); c) alterações emocionais (e.g., medo, cólera, morbilidade psicopatológica); d) alterações na relação com a rede (e.g., isolamento); e) claudicação familiar; e f) luto antecipatório (Cobos, Almendro, Strempel, \& Molina, $2002 \mathrm{a}, \mathrm{b}$ ).

Neste sentido, é uma fase particularmente desafiante para as famílias, com impacto nos seus elementos e relações e, de um modo geral, na qualidade de vida e funcionamento familiar (Forbat, McManus, \& Haraldsdottir, 2012; Kissane \& Bloch, 2002; Rolland, 2005). No entanto, a investigação em cuidados paliativos focada na família é considerada muito incipiente e entende-se como prioritário o estudo dos impactos da doença nos familiares, ao nível das suas necessidades e resposta emocional (Hudson \& Payne, 2011). Posto isto, este estudo visa ampliar o conhecimento acerca da experiência da família face à eminência da morte e tem como principal objetivo compreender o impacto da doença oncológica terminal no sistema familiar em geral e nos seus elementos em particular, atendendo ao contexto de prestação de cuidados: grupo de familiares de doentes internados em unidades de cuidados paliativos (internamento) e o grupo de familiares de doentes a receber cuidados paliativos em casa (domicílio). Mais especificamente, pretendeu-se verificar se existem diferenças entre os dois contextos de prestação de cuidados na: a) importância e na satisfação das necessidades dos familiares; b) experiência individual de luto antecipatório; e c) presença de morbilidade psicopatológica. Finalmente, visou-se d) verificar se a satisfação inadequada das necessidades, a presença de morbilidade psicológica, a experiência de luto antecipatório e o contexto da prestação dos cuidados influenciam a perceção da qualidade de vida familiar. 


\section{METODOLOGIA}

\section{Procedimentos}

O presente estudo encontra-se inserido no projeto de investigação "Necessidades dos Familiares de Doentes Oncológicos Terminais em Cuidados Paliativos", financiado pela Fundação para a Ciência e Tecnologia (SFRH/BD/86178/2012), que tem como objetivo determinar o impacto da doença oncológica terminal no sistema familiar e seus elementos.

Após formalização de pedidos de autorização junto das comissões de ética e conselhos de administração hospitalares, obteve-se autorização para prossecução do estudo em três unidades de cuidados paliativos do centro e sul de Portugal.

A recolha da amostra diferiu consoante o contexto de prestação de cuidados. Em contexto de internamento, o protocolo foi administrado aos familiares numa sala reservada exclusivamente para o efeito. Em contexto domiciliário, o protocolo era administrado em casa dos familiares em dia e hora acordados prévia e telefonicamente. A administração do protocolo, em ambos os casos, foi efetuada pela investigadora principal ou por membros colaboradores da equipa que, antes de o administrar, esclareceu os objetivos principais da investigação, eventuais questões dos familiares e garantiu o anonimato e confidencialidade dos dados. Os participantes preencheram, também, um documento de consentimento informado.

A recolha da amostra decorreu entre Janeiro de 2014 e Janeiro de 2015. Foram atendidos os seguintes critérios de inclusão na amostra: a) ser familiar de um doente oncológico terminal a receber cuidados paliativos; b) ter mais de 18 anos; c) saber ler e escrever; e d) não apresentar psicopatologia não compensada.

\section{Amostra}

A caracterização da amostra encontra-se detalhada na Tabela 1. A amostra é constituída por 40 familiares de doentes oncológicos terminais, que apresentam uma média de idades de 46.47 anos $(D P=17.64)$. Mais especificamente, os familiares de doentes a receber cuidados no domicílio apresentam uma idade média de 48.19 anos $(D P=18.42)$ e os familiares de doentes internados apresentam uma idade média de $44.58(D P=17.03)$. A maioria dos sujeitos são do sexo feminino $(82.5 \%)$, casados (65.0\%), residem na região centro do país $(65.0 \%)$ e não frequentaram o 
ensino superior (80.0\%). A maioria dos familiares são filhos do doente (35.0\%) e são os principais cuidadores (60.0\%).

Quanto ao contexto da prestação de cuidados, 52.5\% dos doentes recebem os cuidados no domicílio e $47.5 \%$ encontram-se internados em unidades de cuidados paliativos.

Com o objetivo de testar a independência das variáveis em função do contexto de prestação de cuidados, realizaram-se testes do Qui-Quadrado (estado civil, região de residência e papel na prestação de cuidados); teste de Fisher, quando os pressupostos ao teste do Qui-Quadrado não foram cumpridos (sexo, habilitações literárias e grau de parentesco) e teste de Mann-Whitney (idade). Relativamente às variáveis sexo, idade, estado civil, habilitações literárias, grau de parentesco e papel na prestação de cuidados, não foram verificadas diferenças na sua distribuição ao nível do contexto de prestação de cuidados. No entanto, relativamente à região de residência, verificou-se um maior número de sujeitos a receber cuidados em internamento na zona centro e um maior número de sujeitos a receber cuidados no domicílio na zona sul do país, $c^{2}(1)=19.49, p<.001$.

Tabela 1

Caracterização da Amostra Total e das Subamostras Domicílio e Internamento

\begin{tabular}{llll}
\hline Características & $\begin{array}{l}\text { Domicílio }(\mathrm{n}=21) \\
\mathrm{n}(\%)\end{array}$ & $\begin{array}{l}\text { Internamento }(\mathrm{n}=19) \\
\mathrm{n}(\%)\end{array}$ & $\begin{array}{l}\text { Total }(\mathrm{N}=40) \\
\mathrm{n}(\%)\end{array}$ \\
\hline $\begin{array}{l}\text { Sexo } \\
\text { Feminino }\end{array}$ & $19(47.5)$ & $14(35.0)$ & $33(82.5)$ \\
Masculino & $2(5.0)$ & $5(12.5)$ & $7(17.5)$ \\
$\begin{array}{l}\text { Estado Civil } \\
\text { Casado/união de facto }\end{array}$ & $15(37.5)$ & $11(27.5)$ & $26(65.0)$ \\
Não casado & & $8(20.0)$ & $14(35.0)$ \\
Região de residência & $6(15.0)$ & & $26(65.0)$ \\
Centro & & $19(47.5)$ & $14(35.0)$ \\
Sul & $7(17.5)$ & - & $32(80.0)$ \\
Habilitações literárias & $14(35.0)$ & & $8(20.0)$ \\
$<$ Ensino superior & $19(47.5)$ & $13(32.5)$ & $14(35.0)$ \\
Ensino superior & $2(5.0)$ & $6(15.0)$ & $10(25.0)$ \\
Grau de parentesco & & & $16(40.0)$ \\
Filho(a) & $5(12.5)$ & $9(22.5)$ & \\
Cônjuge & $5(12.5)$ & $5(12.5)$ & $24(60.0)$ \\
Outro & $11(27.5)$ & $5(12.5)$ & $16(40.0)$ \\
Papel na prestação de & & & \\
cuidados & & $13(32.5)$ & \\
Cuidador principal & $11(27.5)$ & $6(15.0)$ & \\
Não cuidador & $10(25.0)$ & & \\
\hline & & & \\
\hline
\end{tabular}

${ }^{a}$ Solteiro, divorciado e viúvo; ${ }^{\text {b }}$ neto, irmão, sobrinho 


\section{Instrumentos}

Questionário sociodemográfico e de dados complementares: permite a recolha de seis tipos de informação: a) dados sociodemográficos; b) dados familiares; c) dados sobre a prestação de cuidados; d) suporte da rede social pessoal; e) apoio psicológico e espiritual; e f) dados médicos sobre a doença.

Family Inventory of Needs [FIN]: constituído por 20 itens e duas subescalas - Importância das Necessidades e Satisfação das Necessidades -, visa avaliar as necessidades de familiares de doentes oncológicos em fase avançada da doença. As necessidades avaliadas pelo FIN estão relacionadas com o bem-estar do doente (e.g., "Tenho necessidade de estar seguro/a que o melhor cuidado possível está a ser dado ao paciente") e com o suporte psicossocial ao próprio familiar (e.g., "Tenho necessidade de sentir que há esperança”). A subescala Importância das Necessidades é uma escala do tipo Likert de cinco pontos, em que 1 corresponde a "Nada importante" e 5 a "Muito importante". A subescala Importância das Necessidades apresenta uma estrutura unidimensional e o resultado total corresponde à média da importância das 20 necessidades (Kristjanson, Atwood, \& Degner, 1995).

A subescala da Satisfação das Necessidades apresenta três opções de resposta (Satisfeita, Satisfeita parcialmente, Não satisfeita). Esta subescala não apresenta um resultado total, sendo que apenas é feita uma contabilização do número de necessidades satisfeitas, satisfeitas parcialmente e não satisfeitas. O FIN apresenta uma elevada consistência interna tanto na subescala da Importância das Necessidades $(\alpha=$ .92) como na subescala da Satisfação das Necessidades $(\alpha=.96)$ (Fridriksdottir et al., 2006; Kristjanson et al., 1995). Os estudos de adaptação e validação para a população portuguesa estão ainda em curso no âmbito do projeto de doutoramento, no qual este estudo se insere. Para o presente estudo, obtivemos um coeficiente alfa de Cronbach de .91 para os itens que compõem a subescala Importância das Necessidades e de .88 para os itens que compõem a subescala Satisfação das Necessidades.

Marwit-Meuser Caregiver Grief Inventory - Short Form [MMCGI-SF]: constituído por 18 itens, distribuídos por três dimensões (Sobrecarga e Sacrifício Pessoal; Sentimento de Tristeza e Saudade; e Preocupação e Sentimento de Isolamento), avalia a experiência de luto antecipatório dos familiares de sujeitos com doença ameaçadora da vida, numa escala do tipo Likert de cinco pontos. A versão original do instrumento apresenta boas propriedades psicométricas (Marwit \& Meuser, 2005). Os estudos de adaptação e validação para a população portuguesa estão em curso no âmbito do projeto de doutoramento no qual este estudo se insere. Para o presente estudo obteve-se um coeficiente de alfa de Cronbach de .89 para a totalidade dos itens da escala.

Brief Symptom Inventory [BSI]: constituído por 53 itens, distribuídos por nove dimensões (e.g., Depressão, Ansiedade, Somatização), avalia a presença de sintomas 
psicopatológicos numa escala do tipo Likert de cinco pontos. Apresenta, ainda, três índices globais: Índice Geral de Sintomas [IGS]; Total de Sintomas Positivos [TSP]; e Índice de Sintomas Positivos [ISP]. Para este estudo, apenas o ISP foi considerado. De um modo geral, apresenta boas propriedades psicométricas (Canavarro, 2007). Para o presente estudo foi avaliada a consistência interna, na qual obtiveram-se valores de coeficientes alfas de Cronbach entre .59 (Hostilidade) e .89 (Somatização).

Quality of life [QOL]/versão breve (formulário parental): constituído por 20 itens, distribuídos por quatro dimensões (Família, Amigos e Saúde; Tempo; Média e Comunidade; e Bem-estar Financeiro), avalia a qualidade de vida familiar percebida pelo indivíduo numa escala do tipo Likert de cinco pontos. De um modo geral, apresenta boas propriedades psicométricas (Almeida, 2013; Olson \& Barnes, 1982, citado em Simões, 2008). Para o presente estudo, utilizámos, apenas, o resultado total da escala, para o qual obtivemos um alfa de Cronbach de .93.

\section{Análise estatística}

As análises estatísticas foram realizadas com recurso ao software Statistical Package for the Social Sciences (versão 22, IBM SPSS). A existência de diferenças nos dois subgrupos foi averiguada através de testes não paramétricos de diferenças (Mann-Whitney) dada a reduzida dimensão da amostra e cálculo do tamanho do efeito ( $d$ de Cohen). A análise descritiva dos resultados (média e desvio-padrão) foi efetuada com o objetivo de determinar a importância das necessidades, experiência de luto antecipatório e presença de morbilidade psicológica. Para determinar a influência da experiência de luto antecipatório, satisfação das necessidades e o contexto da prestação dos cuidados na perceção da qualidade de vida familiar, recorreu-se a uma regressão linear múltipla.

\section{RESULTADOS}

Comparação da experiência de familiares de doentes terminais em contexto de internamento e domiciliário

\section{Necessidades}

O estudo descritivo e de diferenças foi efetuado ao nível das 20 necessidades avaliadas pelo FIN, uma vez que o instrumento apresenta uma estrutura unifatorial. 
Da análise descritiva dos resultados, ao nível da Importância das Necessidades, verificou-se que todas as necessidades foram cotadas, geralmente, como muito ou extremamente importantes - a média da cotação dos itens oscila entre 3.75 e 4.85 -, verificando-se pouca variabilidade nas respostas. Não se verificaram diferenças estatisticamente significativas entre os dois grupos na subescala Importância das Necessidades, $U=196.50, Z=-.08, p=.94$.

Relativamente à subescala Satisfação das Necessidades, verificaram-se diferenças estatisticamente significativas em três itens. Em contexto domiciliário os familiares consideraram que é menos satisfeita a necessidade de saber factos específicos relativos ao prognóstico do doente (Mean rank $=24.14$ ) do que os familiares de doentes em internamento (Mean Rank = 16.47), $(U=123.00 ; Z=-2.04 ; p=.039)$. A diferença dos resultados apresenta um tamanho do efeito pequeno $(d=0.32)$, (Cohen, 1988). Os familiares de doentes a receber cuidados em casa reportam uma menor satisfação relativamente à necessidade de sentir que há esperança (Mean rank $=25.36$ ), por comparação aos familiares de doentes internados (Mean rank = 13.75), $(U=76.50$; $\mathrm{Z}=-3.37 ; p=.001)$. A diferença dos resultados apresenta um tamanho do efeito médio $(d=0.53)$, (Cohen, 1988). Finalmente, os familiares de doentes em contexto domiciliário reportam uma menor satisfação da necessidade de ter ajuda nos cuidados ao paciente (Mean rank $=24.57)$ por comparação aos familiares de doentes internados (Mean rank = 16.50), $(U=114.00 ; Z=-3.18 ; p=.020)$. A diferença dos resultados apresenta um tamanho do efeito médio $(d=0.50)$, (Cohen, 1988).

\section{Luto Antecipatório}

No que diz respeito à experiência de luto antecipatório, não se verificaram diferenças estatisticamente significativas nos resultados avaliados pelo MMCGI-SF, $U=196.50, Z=-.08, p=.94$. Da análise descritiva dos resultados, verificou-se que quer no contexto de internamento, $M=58.84(D P=15.14)$, quer no contexto de domicílio, $M=59.05(D P=12.89)$, os familiares apresentaram níveis elevados de luto antecipatório.

\section{Morbilidade Psicológica}

Finalmente, quanto à morbilidade psicopatológica não existem diferenças estatisticamente significativas nos resultados do ISP avaliados pelo BSI, nos contextos de internamento e domiciliário, $U=176.00, Z=-.64, p=.54$. Da análise descritiva do ISP, verificou-se que os familiares de doentes internados (ISP = 2.0) e os familiares de doentes no domicílio (ISP $=1.9$ ) apresentam valores superiores ao ponto de corte $(>1.7)$. 
Influência da experiência de luto antecipatório, a satisfação das necessidades e o contexto da prestação dos cuidados na perceção da qualidade de vida familiar

Pretendeu-se determinar a influência do contexto de prestação de cuidados, a satisfação inadequada das necessidades, a experiência de luto antecipatório e a presença de morbilidade psicológica na perceção da qualidade de vida familiar.

Para o efeito, foi estabelecido um modelo de regressão linear múltipla entre as variáveis 'qualidade de vida familiar' (variável dependente) e as variáveis independentes: 'contexto de prestação de cuidados' (internamento e domicílio), 'informação sobre o prognóstico' (satisfação inadequada), 'sentir que há esperança' (satisfação inadequada) e 'ter ajuda nos cuidados ao paciente' (satisfação inadequada), 'nível de luto total' (luto antecipatório, MMCGI-SF) e 'índice geral de sintomas' (morbilidade psicológica, BSI).

Atendeu-se, especificamente, à satisfação inadequada (satisfeita parcialmente ou não satisfeita) das necessidades 'informação sobre o prognóstico', 'sentir que há esperança' e 'ter ajuda nos cuidados ao paciente', por terem sido encontradas diferenças estatisticamente significativas nos resultados das mesmas, nos dois grupos de familiares.

Analisaram-se os pressupostos do modelo, em particular o da distribuição normal, homogeneidade, independência dos erros e diagnóstico de multicolinearidade. Os dois primeiros pressupostos foram validados graficamente e o pressuposto da independência dos erros foi validado com a estatística de Durbin-Watson $(d=1.80)$. Da análise do VIF (1.28 - 1.93) não se identificaram problemas de multicolinearidade.

A regressão linear múltipla permitiu identificar que a satisfação inadequada da necessidade de informação sobre o prognóstico $(\beta=-.24, p=.04)$, o luto antecipatório $(\beta=-.45, p<.001)$ e a morbilidade psicológica $(\beta=-.34, p=.01)$ são preditores significativos da perceção da qualidade de vida familiar. O modelo explica $70.0 \%$ da variabilidade na perceção da qualidade de vida familiar $\left(R^{2}=.70\right)$, (cf. Tabela 2$)$.

Tabela 2

Influência da experiência de luto antecipatório, a satisfação inadequada das necessidades, presença da morbilidade psicológica e o contexto da prestação dos cuidados na perceção da qualidade de vida familiar

\begin{tabular}{lccc}
\hline \multicolumn{1}{c}{ Modelo } & $\mathrm{B}$ & Erro padrão & $\beta$ \\
\hline Constante & 101.75 & 8.63 & \\
Contexto de prestação de cuidados & -1.28 & 4.03 & -.04 \\
"Prognóstico" inadequadamente satisfeita & -7.44 & 3.38 & $-.24^{\star}$ \\
"Esperança" inadequadamente satisfeita & .80 & 3.86 & .03 \\
"Ajuda" inadequadamente satisfeita & -6.93 & 4.22 & -.19 \\
Luto antecipatório & -0.50 & 0.13 & $-.45^{\star * *}$ \\
Morbilidade psicológica & -8.08 & 2.77 & $-.34^{\star *}$ \\
\cline { 2 - 4 } & & $R^{2}=.70(p<.001)$ & \\
\hline
\end{tabular}

${ }^{\star} p<.05 ;{ }^{* *} p<.01 ;{ }^{* *} p<.001$ 


\section{DISCUSSÃO DOS RESULTADOS}

Do estudo de comparação das características sociodemográficas entre os dois contextos verificou-se, ao nível da região de residência, um maior número de sujeitos internados no centro do país e um maior número de sujeitos a receber cuidados em casa no sul do país. Estes resultados podem estar relacionados com o tipo de cuidados oferecidos pelas instituições médicas onde a amostra foi recolhida. De facto, as duas unidades de cuidados paliativos que prestam cuidados em contexto de internamento localizam-se na zona centro do país. Em contrapartida, a unidade de cuidados paliativos que presta cuidados em contexto domiciliário localiza-se na zona sul do país. Estes resultados eram esperados e, de algum modo, incontornáveis, pelo que não invalidaram a prossecução do estudo principal.

$\mathrm{Da}$ análise dos resultados verifica-se que os familiares de doentes a receber cuidados paliativos em contexto de internamento e em casa parecem reportar necessidades idênticas. No entanto, à semelhança de outros estudos (Ventura, Burney, Brooker, Fletcher, \& Ricciardelli, 2014), quando avaliada a satisfação dessas mesmas necessidades pelos profissionais de saúde, verificou-se que os familiares de doentes no domicílio reportam uma menor satisfação das necessidades psicossociais (comunicação, esperança, ajuda nos cuidados) por comparação com as necessidades físicas do doente que, na maioria das vezes, são satisfeitas. Importa sublinhar que, particularmente no contexto domiciliário, a família do doente tende a ser vista como parceira na prestação de cuidados ao doente (Grande et al., 2009). Em contrapartida, o suporte à família no domicílio parece ser, frequentemente, deficiente e inadequado (Bee, Barnes, \& Luker, 2008), tal como os resultados deste estudo apontam.

Em particular, foi possível verificar que os familiares de doentes a receber cuidados paliativos domiciliários reportam uma menor satisfação da necessidade relativa à comunicação do prognóstico (FIN: 'Saber factos específicos relativos ao prognóstico do paciente'), ainda que a diferença dos resultados tenha apresentado um tamanho do efeito pequeno. A comunicação é uma competência básica e essencial em cuidados paliativos e, simultaneamente, uma das mais difíceis para os profissionais de saúde. Comunicar o prognóstico, quando este é de meses, semanas ou dias, é uma das tarefas mais complexas que o médico deve assumir (Querido, Salazar, \& Neto, 2006). Deve fazer-se de forma gradual (Twycross, 2003), embora não se devam dar respostas precisas (Querido et al., 2006), dado que o cancro na fase terminal é, geralmente, difícil de prognosticar, pois a sua progressão é incerta (Twycross, 2003). Trabalhar a questão do prognóstico é uma tarefa morosa e continuada - devem fomentar-se estratégias para lidar com a incerteza, "viver um dia de cada vez" (Twycross, 2003) - pelo que, em contexto de cuidados domiciliários, pode ficar comprometida (e.g., menor disponibilidade de tempo dos profissionais). O princípio "nunca destruir a 
esperança" é, igual e frequentemente, invocado como motivo para não informar o doente-família da gravidade da situação. Opta-se, por isso, e muitas vezes, por ocultar o prognóstico, o que, contrariamente ao pretendido, destrói a esperança do doente e a sua família, quando confrontados com a real gravidade da situação e, por conseguinte, aumenta a ansiedade e/ou desespero (Twycross, 2003).

Verificou-se neste estudo que os familiares de doentes a receber cuidados paliativos em casa reportam uma menor satisfação da necessidade de sentir que há esperança (FIN: 'Sentir que há esperança') comparativamente aos familiares de doentes internados. A diferença destes resultados é representada por um tamanho médio. No contexto de cuidados em fim-de-vida, o conceito de esperança parece assumir-se paradoxalmente (Tanis \& DiNapoli, 2008) e fomentá-la, tal como preconizado na filosofia dos cuidados paliativos (WHO, 2002), constitui-se uma tarefa altamente complexa e desafiante para os profissionais de saúde (Olsman, Leget, OnwuteakaPhilipsen, \& Willems, 2014; Tanis \& DiNapoli, 2008). Deve haver, antes de mais, um reajustamento de expetativas, transformando um objetivo final e irrealista - a esperança da cura - em esperanças alternativas (Twycross, 2003). Falhar a cura não é falhar o doente e, por isso, ao doente-família deve oferecer-se a esperança de encerrar a existência de uma forma apropriada, conferindo um maior sentido à vida que resta viver (Neto, 2006), ao estabelecer-se/trabalhar-se objetivos alternativos (e.g., resolução de assuntos inacabados, (re)encontro com pessoas significativas, conforto na morte), (Twycross, 2003). Sabe-se, ainda, que há a perceção de uma boa qualidade de vida quando as expetativas de um indivíduo são atingidas pela situação atual. Pelo contrário, haverá a perceção de uma menor qualidade de vida quando se verificam discrepâncias entre as expetativas e a situação atual (Moura, 2011). Por isto, se melhorar a qualidade de vida de doentes e familiares é objetivo injuntivo em cuidados paliativos (WHO, 2002), é crucial diminuir o afastamento entre a esperança irrealista do doente-família e aquilo que é possível (Moura, 2011). Saliente-se, ainda, que expetativas irrealistas, a posteriori, defraudadas estão associadas não só a uma diminuição da qualidade de vida (Moura, 2011) naqueles que acompanham o doente, mas também a consequências afetivas e comportamentais negativas, pelo que é fundamental um trabalho criterioso de ajustamento e substituição de expetativas por parte dos profissionais de saúde (Burns, Quinn, Abernethy, \& Currow, 2015), com o objetivo de fomentar e manter a esperança do doente-família (Hawthorn, 2015).

Outro dos nossos resultados indica que os familiares de doentes a receber cuidados em casa reportam uma menor satisfação da necessidade de ter ajuda nos cuidados ao doente (FIN: 'Ter ajuda nos cuidados ao paciente'). A diferença dos resultados nos dois grupos é representada por um tamanho do efeito médio. Em contexto de cuidados paliativos domiciliários, o papel da família no cuidado e acompanhamento ao doente é crítico (Stajduhar \& Cohen, 2009). Aos familiares cabem as tarefas de 
fazer manutenção e gestão dos sintomas do doente (e.g., dor, dispneia, delirium), manuseamento de equipamentos (e.g., cateter, estoma), administração da medicação, limpeza e desinfeção de feridas, nutrição, gestão do transporte do doente e prestação de suporte emocional, espiritual, financeiro e social (Given, Given, \& Sherwood, 2012). Dada a complexidade deste papel, a maioria dos estudos aponta que os cuidadores carecem de ajuda na prestação de cuidados, partilhando a tarefa com os profissionais de saúde e/ou familiares, o que viabiliza o "respiro" do cuidador (Stajduhar \& Cohen, 2009). No entanto, na maioria das vezes, a prestação de cuidados é levada a cabo apenas por um membro da família - cuidador principal - que, em muitos casos, se sente isolado e abandonado pelos restantes membros da família e amigos o que, naturalmente, compromete a partilha das tarefas associadas à prestação de cuidados (Wittenberg-Lyles, Demiris, Oliver, \& Burt, 2011) e o coloca em especial risco de exaustão física e psicológica (Sautter et al., 2014).

Ao nível dos cuidados paliativos domiciliários, a realidade portuguesa está muito aquém (Capelas, 2010) do que é preconizado internacionalmente (EAPC, 2009, 2010): os serviços de cuidados paliativos existentes são em número reduzido para as atuais necessidades do nosso país, bem como o número de profissionais com formação diferenciada nesta área (Capelas, 2009). Este panorama compromete, invariavelmente, o desejável exercício destes cuidados, numa abordagem que se quer holística ao binómio doente-família (WHO, 2002). Por isto, a não resposta das necessidades psicossociais dos familiares de doentes a receber cuidados paliativos em casa poderá estar relacionada com a exaustão das equipas que, atendendo à atual escassez de recursos, priorizam as necessidades físicas do doente, em detrimento das necessidades psicossociais e espirituais do doente-família.

Este estudo também evidenciou que os familiares de doentes oncológicos terminais, independentemente do contexto de prestação de cuidados, apresentam níveis clinicamente relevantes de morbilidade psicológica. O confronto com a proximidade da morte e o cuidar em fim de vida tem um importante impacto no sistema no seu todo (distress familiar), (Carolan, Smith, \& Forbat, 2015), acarretando desafios emocionais complexos aos familiares do doente terminal (Totman, Pistrang, Smith, Hennessey, \& Martin, 2015). Estes tendem a desenvolver níveis preocupantes de ansiedade e depressão (Götze, Brähler, Gansera, Polze, \& Köhler, 2014), acompanhados de uma importante diminuição da qualidade de vida (Yikilkan, Aypak, \& Görpelioglu, 2014). Importa, por isso, monitorizar criteriosamente e, quando necessário, intervir formalmente, no distress do sistema (Carolan et al., 2015) e dos seus elementos, tão cedo quanto possível (Carduff et al., 2016), atendendo à recursividade das respostas emocionais do doente-família, num contexto de eminência de morte. Só deste modo se poderá prevenir o desenvolvimento de distúrbios de depressão, ansiedade e luto complicado post mortem (Kissane \& Bloch, 2002). 
Finalmente, foi possível verificar que, independentemente do contexto de prestação de cuidados, os familiares dos doentes apresentam níveis importantes de luto antecipatório, tal como previsto na literatura (Kissane \& Bloch, 2002; Rolland, 1990). Adicionalmente, verificou-se que a experiência de luto antecipatório, a par da presença de morbilidade psicológica e da satisfação inadequada da necessidade de "informação sobre o prognóstico", é o preditor mais forte da perceção da qualidade de vida familiar, isto é, níveis de luto severo têm repercussões negativas na qualidade de vida familiar. Estes resultados vêm confirmar que o impacto da eminência da morte deve ser entendida numa perspetiva sistémica/familiar (Kissane \& Bloch, 2002; Tercero, 2002), uma vez que a crise da morte eminente se assume como um dos desafios mais dolorosos para todo o sistema familiar (Rolland, 1990; Walsh \& McGoldrick, 2004). O luto antecipatório é uma resposta intra e interpessoal, em que cada elemento do sistema sofre no contexto familiar e a sua resposta influi, necessariamente, na do sistema e vice-versa (Kissane \& Bloch, 2002; Walsh \& McGoldrick, 2004), com repercussões na qualidade de vida e bem-estar familiar. Carece de especial atenção por parte dos profissionais de saúde pois, quando experienciado de forma disfuncional pelo sistema e seus elementos, pode colocar o sistema em risco de dissolução (Tercero, 2002).

\section{Limitações do estudo}

O presente estudo apresenta algumas limitações. Ressaltam-se, desde logo, as características da amostra, em particular, a sua reduzida dimensão e a prevalência de mulheres. Estudos futuros deverão contemplar uma amostra mais ampla e atender a algumas especificidades dos sistemas familiares: familiares que vivem geograficamente distantes do familiar doente (e.g., emigrantes), familiares de doentes oncológicos pediátricos e famílias em contexto de crise económica. Futuramente será, também, relevante compreender a influência do coping e o funcionamento familiar na adaptação individual à doença na fase terminal. Por fim, seria interessante compreender a interinfluência da adaptação da família e adaptação do doente à doença (e.g., resposta emocional) e à eminência da morte (e.g., luto antecipatório).

\section{CONSIDERAÇÕES FINAIS}

Os familiares do doente oncológico terminal são um grupo particularmente vulnerável: apresentam importantes necessidades que carecem de atendimento 
por parte dos profissionais de saúde, sobretudo do foro psicossocial (e.g., suporte emocional); experimentam níveis de luto importantes que devem ser monitorizados, de forma a prevenir o desenvolvimento de lutos complicados post mortem e garantir a adaptação do sistema familiar à doença e à morte; e apresentam níveis clinicamente relevantes de morbilidade psicopatológica.

Destaca-se que o suporte à família, em contexto de cuidados paliativos domiciliários, parece apresentar algumas lacunas, o que vem enfatizar a importância de a integrar e incluir no plano de cuidados. Comunicar e trabalhar a questão do prognóstico do doente, fomentar e manter a esperança, incentivar à partilha das tarefas associadas à prestação de cuidados e, por conseguinte, aliviar o cuidador principal foram questões prioritárias que emergiram neste estudo e que devem ser alvo de particular atenção por parte dos profissionais de saúde que prestam cuidados no domicílio.

Finalmente, conclui-se que, enquanto pilar no acompanhamento e cuidado ao doente, a família tem importantes necessidades que devem ser reconhecidas e satisfeitas pelos profissionais de saúde, a fim de conseguir prosseguir com o seu papel de acompanhar o doente até à sua morte.

\section{REFERÊNCIAS}

Almeida, S. (2013). Escala de qualidade de vida familiar (Quality of Life-QOL): desenvolvimento de uma versão reduzida para a população portuguesa (Dissertação de mestrado não publicada). Faculdade de Psicologia e Ciências da Educação da Universidade de Coimbra, Coimbra.

Bee, P., Barnes, P., \& Luker, K. (2008). A systematic review of informal caregiver's needs and providing home-based end-of life care to people with cancer. Journal of Clinical Nursing, 18(10), 1397-1393. doi: 10.1111/j.1365-2702.2008.02405.x

Burns, E., Quinn, S., Abernethy, A., \& Currow, D. (2015). Caregiver expectations: Predictors of a worse than expected caregiving experience at the end of life. Journal of Pain and Symptom Management, 50(4), 453-461.

Canavarro, M. C. (2007). Inventário de Sintomas Psicopatológicos: Uma revisão crítica dos estudos realizados em Portugal. In M. Simões, C. Machado, M. Gonçalves, \& L. Almeida (eds.), Avaliação psicológica: Instrumentos validados para a população Portuguesa (vol. III, pp. 305-331). Coimbra: Quarteto.

Capelas, M. (2009). Cuidados paliativos: Uma proposta para Portugal. Cadernos de Saúde, 2(1), 51-57.

Capelas, M. (2010). Equipas de cuidados paliativos domiciliários: quantas e onde são necessárias em Portugal. Cadernos de Saúde, 3(2), 21-26.

Carduff, E., Jarvis, A., Highet, G., Finucane, A., Kendall, M., Harrison, N., Greenacre, J., \& Murray, S. (2016). Piloting a new approach in primary care to identify, assess and support carers of people with terminal illnesses: a feasibility study. BMC Family Practice, 17(16). 1-9. doi: $10.1186 / \mathrm{s} 12875-016-0414-2$ 
Carolan, C., Smith, A., \& Forbat, L. (2015). Conceptualising psychological distress in families in palliative care: Findings from a systematic review. Palliative Medicine, 29(7), 605-632. doi: $10.1177 / 0269216315575680$

Cobos, M., Almendro, E., Strempel, P., \& Molina, R. (2002a). La familia en la enfermedad terminal (I). Medicina de Familia, 3(3), 190-199.

Cobos, M., Almendro, E., Strempel, P., \& Molina, R. (2002b). La familia en la enfermedad terminal (II). Medicina de Familia, 3(4), 262-268.

Cohen, J. (1988). Statistical power analysis for the behavioral sciences (2a ed.). New Jersey: Lawrence Erlbaum Associates.

Cooper, J. (2014). Investigating place of death preference among cancer patients and their carers. ANMJ Palliative Care, 21(10), 44.

Direção Geral da Saúde (2014). Portugal - Doenças oncológicas em números - 2014. Retirado do website da Direção Geral da Saúde: https://www.dgs.pt/

Direção Geral de Saúde (2004). Programa nacional de cuidados paliativos. Retirado do website da Direção Geral de Saúde: https://www.dgs.pt/

European Association for Palliative Care (2009). White Paper on standards and norms for hospice and palliative care in Europe: Part 1. Recommendations from the European Association for Palliative Care. European Journal of Palliative Care, 16(6). 278-289.

European Association for Palliative Care (2010). White Paper on standards and norms for hospice and palliative care in Europe: Part 2. Recommendations from the European Association for Palliative Care. European Journal of Palliative Care, 17(1). 22-33.

Ferrel, B., Borneman, T., \& Thai, C. (2009). Family caregiving in hospitals and palliative care units. In P. Hudson, \& S. Payne (eds), Family carers in palliative care. A guide for health and social care professionals (pp. 131-148). New York: Oxford University Press.

Forbat, L., McManus, E., \& Haraldsdottir, E. (2012). Clinical implications for supporting caregivers at the end-of-life: Findings and from a qualitative study. Contemporary Family Therapy, 34(2), 282-292. doi: 10.1007/s10591-012-9194-6.

Fridriksdottir, N., Sigurdardottir, V., \& Gunnarsdottir, S. (2006). Important needs of families in acute and palliative care settings assessed with the Family Inventory of Needs. Palliative Medicine, 20(4), 425-432.

Given, B., Given, C., \& Sherwood, P. (2012). Family and caregiver needs over the course of the cancer trajectory. The Journal of Supportive Oncology, 10(2), 57-63. doi: 10.1016/j.suponc.2011.10.003.

Götze, H., Brähler, E., Gansera, L., Polze, N., \& Köhler, N. (2014). Psychological distress and quality of life of palliative cancer patients and their caring relatives during home care. Supportive Care in Cancer, 22(2), 775-782. doi: 10.1007/s00520-014-2257-5

Grande, G., Stajduhar, K., Aoun, S., Toye, C., Funk, L., Addington-Hall, J., Payne, S., \& Todd, C. (2009). Supporting lay carers in end of life care: Current gaps and future priorities. Palliative Medicine, 23(4), 339-344. doi: 77/0269216309104875

Hawthorn, M. (2015). The importance of communication in sustaining hope at the end of life. British Journal of Nursing, 24(13), 702-705.

Hudson, P., \& Payne, S. (2009). The future of family caregiving: research, social policy and clinical practice. In P. Hudson, \& S. Payne (eds), Family carers in palliative care. A guide for health and social care professionals (pp. 277-303). New York: Oxford University Press.

Instituto Nacional de Estatística (2015). Anuário Estatístico de Portugal 2014 (Edição 2015). Retirado do website do Instituto Nacional de Estatística: https://www.ine.pt/ 
Instituto Nacional de Estatística (2015). Causas de Morte 2013 (Edição 2015). Retirado do website do Instituto Nacional de Estatística: https://www.ine.pt/

Kissane, D., \& Bloch, S. (2002). Family Focused Grief Therapy: A Model of Family-Centered Care during Palliative Care and Bereavement. London, England: Open University Press.

Kristjanson, L., \& Aoun, S. (2004). Palliative care for families: Remembering the hidden patients. Canadian Journal of Psychiatry, 49(6), 359-365.

Marwit, S. J., \& Meuser, T. M. (2005). Development of a short form inventory to assess grief in caregivers of dementia patients. Death Studies, 29(3), 191-205. doi: 10.1080/07481180590916335

Monroe, B., \& Oliviere, D. (2009). Communicating with family carers. In P. Hudson, \& S. Payne (eds.), Family Carers in Palliative Care. A Guide for Health and Social Care Professionals. (pp. 1-20). New York: Oxford University Press.

Moura, C. (2011). A inevitabilidade da morte e o cuidar em fim de vida: Entre a filosofia e a bioética. Lisboa: Coisas de ler.

Neto, I. (2006). Princípios e filosofia dos cuidados paliativos. In A. Barbosa, \& I. Neto (eds.), Manual de Cuidados Paliativos (pp. 295-308). Lisboa: Faculdade de Medicina de Lisboa.

Olsman, E., Leget, C., Onwuteaka-Philipsen, B., \& Willems, D. (2014). Should palliative care patients' hope be truthful, helpful or valuable? An interpretative synthesis of literature describing healthcare professionals' perspectives on hope of palliative care patients. Palliative Medicine, 28(1), 59-70. doi: 10.1177/0269216313482172

Osswald, W. (2013). Sobre a morte e o morrer. Lisboa: Fundação Francisco Manuel dos Santos.

Querido, A., Salazar, H., \& Neto, I. (2006). Comunicação. In A. Barbosa, \& I. Neto (eds), Manual de Cuidados Paliativos (pp. 357-378). Lisboa: Faculdade de Medicina de Lisboa.

Rolland, J. (1984). Toward a psychosocial typology of chronic and life-threatening illness. Family Systems Medicine, 2(3), 245-262.

Rolland, J. (1987). Family illness paradigms: Evolution and significance. Family Systems Medicine, 5(4), 482-503.

Rolland, J. (1990). Anticipatory loss: A family systems developmental framework. Family Process, 29(3), 229-244.

Rolland, J. (2005). Cancer and the family: An integrative model. Cancer, 104(S11), 2584-2595.

Sautter, J., Tulsky, J., Johnson, K., Olsen, M., Burton-Chase, A., Lindquist, J., Zimmerman, S., \& Steinhauser, K. (2014). Caregiver experience during advanced chronic illness and last year of life. Journal of the American Geriatrics Society, 32(6), 1082-1090.

Siegel, R., Miller, K., \& Jemal, A. (2016). Cancer statistics, 2016. CA: A Cancer Journal for Clinicians, 66(1), 7-30. doi: 10.3322/caac.21332.

Simões, J. (2008). Qualidade de vida: estudo de validação para a população portuguesa (Dissertação de mestrado não publicada). Faculdade de Psicologia e Ciências da Educação, Universidade de Coimbra. Coimbra.

Simões, M. (2014). O cancro. Lisboa: Relógio D’Água Editores.

Stajduhar, K., \& Cohen, R. (2009). Family caregiving in the home. In P. Hudson, \& S. Payne (eds.), Family carers in palliative care. A guide for health and social care professionals. (pp. 149-168). New York: Oxford University Press.

Tanis, S., \& DiNapoli, P. (2008). Paradox of hope in patients receiving palliative care: A concept analysis. International Journal for Human Caring, 12(1), 50-54.

Tercero, R. (2002). Duelo Familiar. Sistemas Familiares, 18(1-2), 48-61.

Totman, J., Pistrang, N., Smith, S., Hennessey, S. \& Martin, J. (2015). 'You only have one chance to get it right': A qualitative study of relatives' experiences of caring at home for a family member with terminal cancer. Palliative Medicine, 29(6). 96-507. doi: 0.1177/0269216314566840 
Twycross, R. (2003). Cuidados paliativos (2a ed.). Lisboa: Climepsi.

Ventura, A., Burney, S., Brooker, J., Fletcher, J., \& Ricciardelli, L. (2014). Home-based palliative care: A systematic literature review of the self-reported unmet needs of patients and carers. Palliative Medicine, 28(5), 391-402. doi: 0.1177/0269216313511141

Walsh, F., \& McGoldrick (2004). Loss and the family: A systemic perspective. In F. Walsh, \& M. McGoldrick (eds.), Living beyond the loss. Death in the family (2a ed., pp. 3-26). New York: W. W. Norton \& Company.

Wittenberg-Lyles, E., Demiris, G., Oliver, D., \& Burt, S. (2011). Reciprocal suffering: Caregiver concerns during hospice care. Journal of Pain and Symptom Management, 41(2), 383-393. doi: 10.1016/j.jpainsymman.2010.04.026.

World Health Organization (2002). National cancer control programmes: Policies and managerial ( $2^{\text {nd }}$ ed.). Geneva: World Health Organization.

World Health Organization (WHO) (2015). Cancer. Fact sheet $n^{\circ} 297$. Retirado do website da World Health Organization: http://www.who.int/mediacentre/factsheets/fs297/en/ 